\title{
Chiral Fermion Field on Random Lattices
}

\author{
T. W. Chiu ${ }^{\mathrm{a} *}$ \\ aPhysics Department, National Taiwan University, \\ Taipei, Taiwan 106, R.O.C.
}

The fermion doubling problem is discussed from the viewpoint of functional integration. The measure provided by any single lattice is not sufficent to yield solutions to agree with the continu um field theory. The approach of using an ensemble of random-block lattices to provide the proper measure is outlined. Some salient features of randomization are discussed.

It is next to impossible to formulate a chiral fermion theory on a single hypercubical lattice such that it has the desirable continuum limit. These diffuculties are summarized in the No-Go theorem of Nielson and Ninomiya [1]. Although the original proof of this theorem was essentially for the free fermion on a hypercubical lattice, it is widely believed that the theorem should also hold when interactions are turned on, and also for any single ( regular or random ) lattice. However, the No-Go theorem never limits us to recover the correct continuum field theory by using an ensemble of random lattices. It should be emphasized that ensemble averaging is a very non-trivial process which attempts to incorporate all functional integrations which must be partially omitted by any single lattice. Such omissions do not cause any serious problems in bosonic field theories, but catastrophe in fermionic ones. Field theories on a random lattice were first formulated by Christ, Friedberg and Lee [2] in 1982. Their motivations were to preserve the translational and rotational symmetry which are explicitly broken by the hypercubical lattice. Their construction of a $2 \mathrm{D}$ random lattice is exactly the same as the Voronoi-Delaunay prescription which can be generalized to higher dimensions. In 1986, Pang and Ren [3], and independently Espriu, Gross, Rakow and Wheater [4], using an ensemble of several hundreds of $2 \mathrm{D}$ random lattices, demonstrated that for the free massive fermion theory, the ensemble-averaged fermion propagators agree

\footnotetext{
"Research supported by National Science Council, R.O.C. under the grant NSC83-0208-MO02-024.
}

with the continuum field theory. In 1988, Ren [5] studied the massless fermion field in an external abelian gauge field on $2 \mathrm{D}$ random lattices and found that the ensemble-averaged fermion propagators agree with the continuum field theory but the composite boson propagators ( even after point-splitting ) are not in agreement with the continuum theory. Almost at the same time, Perantonis and Wheater [6] stuided a Yukawa coupling model and the massive Thirring model on $2 \mathrm{D}$ random lattices in weak coupling perturbation theory. They found that the ultraviolet divergent parts do not exhibit the same dependence on the tree level mass and thus causes a fine tuning problem. These unfavorable results [5] [6] of interacting fermion field theories have decreased further studies of fermion field on CFL random lattices. However, we think that randomization is a useful procedure and thus to understand why CFL random lattices do not work would help us to discover the correct randomization for lattice chiral fermion.

A field theory on a random lattice has two related aspects of randomness : the random geometry and the random couplings (weights) resulting from the random geometry. The random geometry mainly provides a proper measure for the functional integrations. The random coupling between any two neighbouring fields is the weight assigned according to a certain prescription which takes into account of the random geometry in the vicinity of these two neighbouring sites. The random couplings are short-ranged interactions and thus have little effects on tree di- 
agrams but strongly affect the higher-order ones. From this point of view, the results of [5] and [6] may have indicated that in a CFL random lattice, the random couplings are too strong and produce a large number of low-lying states whose effects are persistent in all higher order diagrams. For the free fermion propagator at large distances, the most relevant randomness is due to the random geometry. In the equal-weight limit ( setting all random couplings to be the same), the random geometry alone would give the ensemble-averaged fermion propagator to agree with the continuum field theory. On the other hand, if we fix the geometry to a regular lattice and introduce random couplings between neighbouring fields, one could not obtain the correct fermion propagator for any distributions of random couplings. The reason is that the most important ingredient, the random geometry is absent and therefore some of the important functional integrations must be omitted. Randomization is a very tricky procedure. It is by no means that any randomization scheme would work for the lattice fermion.

In order to understand the difficulties of lattice fermion and to search for the correct randomization scheme, it is instructive to consider the simplest case, massless naive fermion on a Ddimensional hypercubical lattice. The action is

$$
A_{\text {reg }}=\sum_{\mu=1}^{D} \sum_{i} a^{D} \frac{\bar{\psi}(i) \gamma^{\mu}[\psi(i+\hat{\mu})-\psi(i-\hat{\mu})]}{2 a}
$$

where $\bar{\psi}(i)$ and $\psi(i)$ are two independent fermion fields at site $i$, and $a$ is the lattice spacing. The fermion propagator from the site $y=$ $\left(j_{1}, j_{2}, \cdots, j_{D}\right) a$ to the site $x=\left(i_{1}, i_{2}, \cdots, i_{D}\right) a$ is

$S_{F}(x, y)=<\psi(x) \bar{\psi}(y)>=\sum_{\mu=1}^{D} \gamma_{\mu} S_{\mu}(x, y)$

where $S_{\mu}(x, y)$ is very different from its counterpart $S_{\mu}^{c}(x, y)$ in continuum field theory due to an extra discrete factor which is either zero or $2^{D}$. This discrete factor is

$$
T_{\mu}(x, y)=\left[1-(-1)^{n_{\mu}}\right] \prod_{\nu \neq \mu}^{D}\left[1+(-1)^{n_{\nu}}\right]
$$

where $n_{\lambda} \equiv i_{\lambda}-j_{\lambda}, \lambda=1, \cdots, D$. Then $S_{\mu}(x, y)$ can be written as

$S_{\mu}(x, y)=R_{\mu}(x, y) T_{\mu}(x, y)$

where

$R_{\mu}(x, y) \stackrel{a \rightarrow 0}{\longrightarrow} S_{\mu}^{c}(x, y)$

and $S_{\mu}^{c}(x, y)$ is the component of the fermion propagator $S_{F}^{c}(x, y)=\sum_{\mu=1}^{D} \gamma_{\mu} S_{\mu}^{c}(x, y)$ in continuum. The problem of lattice fermion on a D-dimensional hypercubical lattice is essentially due to the discrete factor $T_{\mu}(x, y)$ associated with $S_{\mu}(x, y)$. For example, consider $x$ and $y$ both lying on the body diagonal (i.e., $i_{1}=i_{2}=\cdots=i_{D}$ and $\left.j_{1}=j_{2}=\cdots=j_{D}\right)$, then $S_{\mu}(x, y)=0$ identically for $\mu=1, \cdots, D$ ! If we view the propagator as the transition amplitude between $x$ and $y$, then the hypercubical lattice only provides those paths such that the sum of transition amplitudes along all possible paths connecting $x$ and $y$ cancel each other completely ! In general, there is no any single lattice ( random or regular) which can provide the paths such that it can yield the correct fermion propagator. For the hypercubical lattice, the discrete factor $T_{\mu}(x, y)$ is quite general, which exists even in the presence of background fields interacting with the fermion field. Expanding $T_{\mu}(x, y)$ in eq. (3) yields $2^{D}$ terms of which only the constant term 1 has the correct continuum limit. The other $2^{D}-1$ terms can be interpreted, in the momentum space, as the contributions of the $2^{D}-1$ doubled modes near the momentum regions $\otimes_{\lambda=1}^{D}\left(0, \frac{\pi}{a}\right)_{\lambda}$ excluding the origin. On a CFL random lattice, in general, the fermion propagators do not have the discrete factors $T_{\mu}(x, y)$ as those on a regular lattice, but they still disagree with the continuum propagators. Therefore we cannot ascribe the discrepancies to the doubled modes. The existence of the fermion problem in any single lattice, independent of the lattice structure, indicates that the cause is not due to the doubled modes but the deficiency of the measure provided by a single lattice. If we employ an ensemble of Random-Block Lattices (RBL) [7] [8], the probability distribution of sites along each direction is an indepen- 
dent Poisson distribution

$P\left(\left|x_{\lambda}-y_{\lambda}\right|, n_{\lambda}\right)=\frac{1}{n_{\lambda} !}\left(\frac{\left|x_{\lambda}-y_{\lambda}\right|}{a}\right)^{n_{\lambda}} e^{-\frac{\left|x_{\lambda}-y_{\lambda}\right|}{a}}$

Then after ensemble averaging over $R B L$, the fermion propagator becomes

$<S_{F}(x, y)>=\sum_{\mu=1}^{D} \gamma_{\mu} R_{\mu}(x, y)<T_{\mu}(x, y)>$

where

$$
\begin{aligned}
& <T_{\mu}(x, y)>=\sum_{\lambda=1}^{D} \sum_{n_{\lambda}} \prod_{\sigma=1}^{D} P\left(\left|x_{\sigma}-y_{\sigma}\right|, n_{\sigma}\right) T_{\mu}(x, y) \\
& =\left[1+e^{-\frac{2\left|x_{\mu}-y_{\mu}\right|}{a}}\right] \prod_{\nu \neq \mu}\left[1-e^{-\frac{2\left|x_{\nu}-y_{\nu}\right|}{a}}\right] \stackrel{a \rightarrow 0}{\longrightarrow} 1
\end{aligned}
$$

Hence, in the continuum limit, the ensembleaveraged fermion propagator

$<S_{F}(x, y)>\stackrel{a \rightarrow 0}{\longrightarrow} \sum_{\mu=1}^{D} \gamma_{\mu} S_{\mu}^{c}(x, y)$

agrees with the continuum field theory. This proves that the massless fermion action on RBL

$A_{r b l}=\sum_{\mu=1}^{D} \sum_{i} \omega_{i} K_{i}^{\mu} \bar{\psi}(i) \gamma^{\mu}[\psi(i+\hat{\mu})-\psi(i-\hat{\mu})]$

in the equal-weight limit $\left(\omega_{i}=a^{D}, K_{i}^{\mu}=\frac{1}{2 a}\right)$, gives the ensemble-averaged fermion propagator in agreement with the continuum field thoery. When the random weights $\left(\omega_{i}, K_{i}^{\mu}\right)$ are turned on, the fermion propagator on a RBL still exhibit the discrete factor $T_{\mu}(x, y)$. Furthermore these short-ranged interactions help to yield better results than those in the equal-weight limit, especially for propagators at smaller distances. Although this statement has not been proved analytically, it is supported by a large amount of numerical results. When a background gauge field is introduced, the fermion propagator on a RBL still exhibit the discrete factor $T_{\mu}(x, y)$. It can be shown that in the equal-weight limit, the fermion propagator, the axial anomaly, the gauge invariant currents and current-current correlations all agree with the continuum field theory. Some numerical results for $\mathrm{D}=2$ were described in [9]. Since the discrete factor $T_{\mu}(x, y)$ persists when a background gauge field is turned on, then on a given $\mathrm{RBL}$, the locations of the zeros of $T_{\mu}(x, y)$ must be the same for all gauge field configurations. This implies that the RBL scheme would work for the quenched approximation. The most difficult case of dynamical fermions interacting with gauge fields must be studied by fully dynamical calculations. Other studies such as measuring the corrections to the fermion determinant due to a background gauge field cannot serve our purpose. A fully dynamical calculation using hybrid monte carlo algorithm is now in progress.

Finally we would like to remark that on a CFL random lattice, the discrete factor $T_{\mu}(x, y)$ is absent and the above analysis cannot be applied. Therefore it is not obvious how the ensembleaveraging could give the correct continuum limit. In fact, for a massless fermion field in a background abelian gauge field in 2D, we could not obtain the correct axial anomaly or current-current correlations even after ensemble averaging over 409,600 CFL random lattices!

\section{REFERENCES}

1. H.B. Nielsen and N. Ninomiya, Nucl. Phys. B185, 20 (1981); B193, 173 (1981).

2. N.H. Christ, R. Friedberg and T.D. Lee, Nucl. Phys. B202 (1982) 89; B210 [FS6] (1982) 310; B210 [FS6] (1982) 337.

3. Y. Pang and H.C. Ren, Phys. Lett. B172 (1986) 392.

4. D. Espriu, M. Gross, P.E.L. Rakow and J.F. Wheater, Nucl. Phys. B275 [FS17] (1986) 39.

5. H.C. Ren, Nucl. Phys. B300 [FS22] (1988) 531 .

6. S.J. Perantonis and J.F. Wheater, Nucl. Phys. B295 (1988) 443.

7. T.W. Chiu, Phys. Lett. B 206 (1988) 510.

8. T.W. Chiu, "Field Theory on the RandomBlock Lattice", in Proceedings of the 25th International Conference on High Energy Physics (World Scientific, 1991 ).

9. T.W. Chiu, Nucl. Phys. B ( Proc. Suppl.) 34 (1994); " Massless Fermion Field on 2D Random-Block Lattice ", NTUTH-93-16, 1994. 